\title{
I-SceI-mediated double-strand DNA breaks stimulate efficient gene targeting in the industrial fungus Trichoderma reesei
}

\author{
Jean Paul Ouedraogo ${ }^{1}$ - Mark Arentshorst ${ }^{1} \cdot$ Igor Nikolaev $^{2}$ • \\ Sharief Barends ${ }^{2}$ - Arthur F. J. Ram ${ }^{1}$
}

Received: 5 May 2015 / Revised: 3 July 2015 / Accepted: 7 July 2015 / Published online: 15 August 2015

(C) The Author(s) 2015. This article is published with open access at Springerlink.com

\begin{abstract}
Targeted integration of expression cassettes for enzyme production in industrial microorganisms is desirable especially when enzyme variants are screened for improved enzymatic properties. However, currently used methods for targeted integration are inefficient and result in low transformation frequencies. In this study, we expressed the Saccharomyces cerevisiae I-SceI meganuclease to generate double-strand breaks at a defined locus in the Trichoderma reesei genome. We showed that the double-strand DNA breaks mediated by I-SceI can be efficiently repaired when an exogenous DNA cassette flanked by regions homologous to the I-SceI landing locus was added during transformation. Transformation efficiencies increased approximately sixfold compared to control transformation. Analysis of the transformants obtained via I-SceI-mediated gene targeting showed that about two thirds of the transformants resulted from a homologous recombination event at the predetermined locus. Counter selection of the transformants for the loss of the pyr $G$ marker upon integration of the DNA cassette showed that almost all of the clones contained the cassette at the predetermined locus. Analysis of independently obtained transformants using targeted integration of a glucoamylase
\end{abstract}

Electronic supplementary material The online version of this article (doi:10.1007/s00253-015-6829-1) contains supplementary material, which is available to authorized users.

Arthur F. J. Ram

A.F.J.Ram@biology.leidenuniv.nl

1 Molecular Microbiology and Biotechnology, Institute of Biology Leiden, Kluyver Centre for Genomics of Industrial Fermentation, Leiden University, Sylviusweg 72, 2333 BE Leiden, The Netherlands

2 Dupont Industrial Biosciences, Archimedesweg 30, 2333 CN Leiden, The Netherlands expression cassette demonstrated that glucoamylase production among the transformants was high and showing limited variation. In conclusion, the gene targeting system developed in this study significantly increases transformation efficiency as well as homologous recombination efficiency and omits the use of $\Delta k u 70$ strains. It is also suitable for high-throughput screening of enzyme variants or gene libraries in $T$. reesei.

Keywords Meganuclease - DNA repair - Counter selection · Targeted integration $\cdot$ Hypocrea jecorina

\section{Introduction}

Trichoderma reesei (teleomorph Hypocrea jecorina) can secrete large amounts of extracellular protein (up to $100 \mathrm{~g} / \mathrm{L}$ ), which makes it a paradigm host for homologous and heterologous protein production (Anderson et al. 2013; Schuster and Schmoll 2010). To improve production, activity or other properties of industrially interesting enzymes, both random and systematic approaches to generate enzyme variants are employed (Adrio and Demain 2014; Turner 2009). To simplify comparison among enzyme variants or different homologues expressed in filamentous fungi and, in particular $T$. reesei, it is highly desirable that in order to ensure an identical genetic environment, the corresponding DNA constructs are integrated at a defined locus in the genome. Random integration of expression cassettes often leads to significant variation in production levels caused by differences in copy number and/or sites of integration. Therefore, it has been attempted to increase the efficiency of gene targeting in $T$. reesei by several means, such as by increasing the size of the region homologous to the target locus (Catalano et al. 2011) or by developing strains that are deficient in non-homologous end joining (NHEJ) (Catalano et al. 2011; Guangtao et al. 2009). 
However, even though the efficiency of homologous integration events is tremendously increased in strains deficient in NHEJ, it remains highly locus-dependent (Schuster et al. 2012). Transformation frequencies with NHEJ-deficient strains are often low, making it difficult and laborious to generate enough mutants for library screening. Consequently, there is still a need to develop alternative methods for efficient gene targeting into the genome of $T$. reesei that lead to high homogeneity in protein expression among transformants. Another limiting factor associated with $T$. reesei transformation is a relative low frequency of stable, DNA integration events. A substantial number of primary transformants (30$50 \%$ ) are abortive ones, in which a plasmid is only transiently expressed and not integrated into the genome.

In this study, we present an approach that not only increases the number of stable transformants in $T$. reesei up to sixfold but also increases the efficiency of targeted integration of the gene of interest. The approach is based on stimulating the formation of a DNA double-strand break (DSB) at a specific locus of the genome, which is subsequently efficiently repaired by the receiving organism to maintain its genomic integrity and survival (Bollag et al. 1989; Szostak et al. 1983). Eukaryotic cells repair DSBs either by the NHEJ pathway or via homology directed repair (HDR) with homologous sequences flanking the DSBs or any other available homologous template, including exogenous donor sequences (Cahill et al. 2006). Genomic DSBs can occur either naturally or be induced artificially. It has been shown that DSBs can be specifically induced in eukaryotic genomes by using the yeast ISceI endonuclease (Arazoe et al. 2014; Choulika et al. 1995; Kuijpers et al. 2013; Puchta et al. 1993; Rouet et al. 1994). ISceI is a mitochondrial homing endonuclease encoded by the Saccharomyces cerevisiase mitochondrial genome and recognizes a 18 -basepair-long DNA sequence (5'TAGGGATAACAGGGTAAT-3') (Monteilhet et al. 1990; Plessis et al. 1992; Fairhead and Dujon 1993). Since the recognition site is rather long and specific, I-SceI recognition sites are absent in most eukaryotic genomes, including the T. reesei genome. Expression of the endonuclease I-SceI has been previously demonstrated to efficiently induce DSBs at ISceI sites inserted at specific sites in prokaryotic cells (Meddows et al. 2005) and also in several eukaryotes, including mammalian cells (Choulika et al. 1995), plants (Puchta et al. 1993) and unicellular eukaryotes (Glover and Horn 2009). In filamentous fungi, I-SceI-mediated DSBs have been recently described in Pyricularia oryzae (Arazoe et al. 2014), a fungus belonging to the class of Sordariomycetes, to which $T$. reesei also belongs. In the present report, we have introduced I-SceI recognition sites into the genome of $T$. reesei and have investigated the effect of DSBs mediated by heterologous expression of I-SceI on transformation efficiency, on frequency of targeted integration of a reporter gene and on the expression levels of the reporter protein. We have also shown that about two thirds of the transformants integrated a reporter $T$. reesei glucoamylase gene at a pre-assigned locus and expressed it uniformly.

\section{Materials and methods}

\section{Strains and cultivation conditions}

T. reesei strain RL-P37 $\Delta$ cbhIpyrG-26 (from DuPont Industrial Bioscience division, Leiden, The Netherlands) was used as a parental strain throughout the study. RL-P37 $\Delta$ cbhIpyrG-26 was developed from the wild-type T. reesei strain, QM6a, via several classical mutagenesis steps and was modified to delete the gene encoding cellobiohydrolase 1 (Cbh1) and inactivate the pyr 4 gene. T. reesei strains derived from the parental strain and used in this study are listed in Table 1. T. reesei strains were maintained on Trichoderma agar minimal medium (TrMM) containing $15 \mathrm{~g} / \mathrm{L} \mathrm{KH}_{2} \mathrm{PO}_{4}, 5 \mathrm{~g} / \mathrm{L}$ $\left(\mathrm{NH}_{4}\right)_{2} \mathrm{SO}_{4}, 20 \mathrm{~g} / \mathrm{L}$ carbon source (glucose or lactose), $0.6 \mathrm{~g} /$ $\mathrm{L} \mathrm{MgSO}_{4} \cdot 7 \mathrm{H}_{2} \mathrm{O}, 0.6 \mathrm{~g} / \mathrm{L}(\mathrm{mM}) \mathrm{CaCl}_{2} \cdot 2 \mathrm{H}_{2} \mathrm{O}, 1 \mathrm{~mL} / \mathrm{L}$ trace element solution $\left(175 \mathrm{~g} / \mathrm{L} \mathrm{C}_{6} \mathrm{H}_{8} \mathrm{O}_{7}, 200 \mathrm{~g} / \mathrm{L} \mathrm{FeSO}_{4} \cdot 7 \mathrm{H}_{2} \mathrm{O}\right.$, $16 \mathrm{~g} / \mathrm{L} \mathrm{ZnSO}_{4} \cdot 7 \mathrm{H}_{2} \mathrm{O}, 3.2 \mathrm{~g} / \mathrm{L} \mathrm{CuSO}_{4} \cdot 5 \mathrm{H}_{2} \mathrm{O}, 1.4 \mathrm{~g} / \mathrm{L} \mathrm{MnSO}_{4}$. $\mathrm{H}_{2} \mathrm{O}$ and $0.8 \mathrm{~g} / \mathrm{L} \mathrm{H}_{3} \mathrm{BO}_{3}$ ) and $2 \%$ agar and set at $\mathrm{pH} 4.8$. When required, the medium was supplemented with $10 \mathrm{mM}$ uridine or $10 \mathrm{mM}$ acetamide instead of $\left(\mathrm{NH}_{4}\right)_{2} \mathrm{SO}_{4}$ (for $a m d S$ selection) or $5 \mu \mathrm{g} / \mathrm{ml}$ of chlorimuron ethyl (for acetolactate synthase alS gene selection) (Bower et al. 2012). T. reesei strains were routinely cultivated at $30{ }^{\circ} \mathrm{C}$ in the presence of light.

T. reesei was transformed using the polyethylene glycol (PEG)-mediated protoplast transformation protocol described by Penttilä et al. (1987) with slight modifications. Fiftymillilitre cultures inoculated with $5 \times 10^{8}$ conidia were grown in the dark at $30^{\circ} \mathrm{C}$ and $200 \mathrm{rpm}$ for $12-20 \mathrm{~h}$. A total of $675 \mathrm{mg}$ lysing enzyme (Sigma-Aldrich, Zwijndrecht, The Netherlands) were dissolved in $15 \mathrm{~mL}$ of $1.2 \mathrm{M}$ $\mathrm{MgSO}_{4}-10 \mathrm{mM}$ sodium phosphate buffer, pH 5.8. Protoplasting was performed at $25{ }^{\circ} \mathrm{C}$ and $90 \mathrm{rpm}$ and was verified every $30 \mathrm{~min}$ by microscopy. Two hundred microlitres of protoplast suspension was mixed with 5$10 \mu \mathrm{g}$ of DNA and $2 \mathrm{~mL}$ of freshly made PEG buffer. Stable transformants were obtained by streaking on TrMM plates containing the required selection pressure, for two successive rounds. Single colonies obtained after double streaking were selected for sporulation and further analysis.

T. reesei liquid cultures were grown in 24-well plates configured such as to release lactose from a solid, porous matrix. Each well contained $1.25 \mathrm{~mL}$ of an NREL medium $(9 \mathrm{~g} / \mathrm{L}$ casamino acids, $5 \mathrm{~g} / \mathrm{L}\left(\mathrm{NH}_{4}\right)_{2} \mathrm{SO}_{4}, 4.5 \mathrm{~g} / \mathrm{L} \mathrm{KH}_{2} \mathrm{PO} 4,1 \mathrm{~g} / \mathrm{L}$ $\mathrm{MgSO}_{4} \cdot 7 \mathrm{H}_{2} \mathrm{O}, 1 \mathrm{~g} / \mathrm{L} \mathrm{CaCl}{ }_{2} \cdot 2 \mathrm{H}_{2} \mathrm{O}, 33 \mathrm{~g} / \mathrm{L}$ PIPPS buffer, at $\mathrm{pH} 5.5,0.25 \%$ T. reesei trace elements, as described above). 
Table 1 Fungal strains and plasmids used in this study

\begin{tabular}{|c|c|c|}
\hline & Genotype or description & Source or reference \\
\hline \multicolumn{3}{|l|}{ Fungal strains } \\
\hline QM6a & Wild-type strain & Anderson et al. 2013 \\
\hline P37 3 cbhIpyrG-26 & pyr $4^{-}$and $P c b h \Gamma^{-}$ & $\begin{array}{l}\text { DuPont bioscience. Leiden, } \\
\text { The Netherlands }\end{array}$ \\
\hline JP7.7 & P37 3 cbhIpyrG-26 with I-SceI restriction site cassette integrated at $c b h 2$ locus & This study \\
\hline JP7.7_pTTT & JP7.7+ pTTT & This study \\
\hline JP7.7.12 & JP7.7+ pTTT-ISceI & This study \\
\hline JP7.7.14 & JP7.7+ pTTT-ISceI & This study \\
\hline \multicolumn{3}{|l|}{ Plasmids } \\
\hline pBJP6 & Carries a I-SceI restriction site cassette & This study \\
\hline pCRpyrGAN & Contains the full gene of $A$. nidulans pyrG & This study \\
\hline pTTT & $c b h I$ promoter and $a m d S$ selection & DuPont bioscience \\
\hline pTTT-ISceI & I-SceI under control of the inducible $c b h 1$ promoter with $a m d S$ selection & This study \\
\hline Ptrex6gGA/wt & Carries the $T$. reesei glucoamylase gene & DuPont bioscience \\
\hline pJP8 & $\begin{array}{l}\text { Carries a } T \text {. reesei glucoamylase cassette with homologous regions of the } \\
\text { I-SceI landing sites cassette ( } p B J P 6)\end{array}$ & This study \\
\hline
\end{tabular}

Escherichia coli DH5 $\alpha$ strain was used for plasmid construction and propagation using standard techniques.

\section{DNA manipulations and molecular analyses}

Genomic DNA extraction of T. reesei, diagnostic PCR and Southern blot analysis were performed as previously described (Meyer et al. 2010). Restriction enzymes and DNA dephosphorylation and ligation kits were obtained from Invitrogen (Bleiswijk, The Netherlands) or Thermo Fisher (Leusden, The Netherlands) and used according to the instructions of the manufacturer. Sequencing was performed by Macrogen (Amsterdam, The Netherlands).

\section{Construction of the I-SceI restriction site cassette}

The plasmid pBJP6 with two I-SceI restriction sites was made as follows (Fig. S1 in the Supplementary Material). The 5'UTR $c b h 2, P c b h I$ and $T c b h 2$ were amplified from genomic DNA of QM6a with primers GSP1 and GSP2, PP1 and PP2, and GSP3 and GSP4, respectively (Table 2). The PCR fragments were fused using Phusion DNA polymerase (Thermo Fisher,

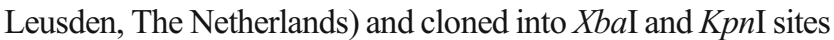
of pBluescriptSK $(+)$ (Stratagene, CA, USA) resulting in the plasmid pBJP4 (Fig. S1 in the Supplementary Material). DNA sequences encoding for N-terminally and C-terminally truncated green fluorescent protein (GFP) with $0.6-\mathrm{kbp}$ overlapping sequences were synthetically ordered based on the GFP sequence of Ptilosarcus sp. (Geneart, Regensburg, Germany) and cloned into the NdeI site of pBJP4, resulting in the plasmid pBJP5. Restriction analysis was performed to ensure that the N-terminal sequence of GFP which contains the start codon is inserted properly after the promoter $c b h I$. The final pBPJ6 plasmid was obtained by amplifying the pyrG marker of Aspergillus nidulans flanked by two I-SceI sites with the primers FwpGAN-ISceIPmeI and RevpGANISceIPmeI from the plasmid pCRpyrGAN and cloned into the PmeI site of pBJP5. pCRpyrGAN is a pCR-Topo Bluntbased vector with the kanamycin resistance marker containing a PCR-amplified 1.7-kb genomic DNA fragment of the A. nidulans pyrG gene. Primers FwpGAN-ISceIPmeI and RevpGAN-ISceIPmeI were designed to incorporate the I-SceI restriction sites and the PmeI sites downstream and upstream of the pyr $G$ marker during PCR amplification (see primers sequences in Table 2). The sequence of the pBJP6 plasmid was confirmed by restriction analysis and sequencing.

\section{Construction of the I-SceI expression vector}

A codon-optimized gene coding for the Saccharomyces cerevisiae I-SceI sequence (SGD ID S000007279) was synthesized for expression in T. reesei (Geneart, Regensburg, Germany). The nucleotide sequence encoding I-SceI has been deposited in GenBank under accession number KR584660. To construct a I-SceI expression vector, the I-SceI gene was cloned via a Gateway recombination into the telomeric plasmid pTTT (Aehle et al. 2011), where I-SceI expression was driven by the T. reesei cbhI-inducible promoter. A I-SceI variant in which we included the nuclear localization signal (NLS) (MATPSSVASS SSRDQVQRIH RVTRENRHLW YQLTVLQQPE RARACGSG) of the T. reesei Velvet protein (JGI ID 122284) was constructed and tested in parallel. To 
Table 2 List of primers used in this study

\begin{tabular}{|c|c|c|c|}
\hline Primer name & $\mathrm{f} / \mathrm{r}$ & Sequence ( $5^{\prime}$ to $3^{\prime}$ oriented) & Template \\
\hline GSP1 $(X b a \mathrm{I})$ & $\mathrm{f}$ & TCTAGAGGCTGTGCATTTCCGTTCTC & gDNA QM6a \\
\hline GSP2 & $\mathrm{r}$ & TGGTTACGGCAACAAACCTG & gDNA QM6a \\
\hline PP1 & $\mathrm{f}$ & CAGGTTTGTTGCCGTAACCAATTTGCCTGCTTGACCGACTG & gDNA QM6a \\
\hline PP2 & $\mathrm{r}$ & GGAACGATGGGTTTGCGTCCATATGGGGTAAGTCACTTACGGCAGC & gDNA QM6a \\
\hline GSP3 (NdeI) & $\mathrm{f}$ & CCATATGGACGCAAACCCATCGTTCC & gDNA QM6a \\
\hline GSP4 $(K p n \mathrm{I})$ & $\mathrm{r}$ & GGTACCGGTTCACCGCCTTATGTGAG & gDNA QM6a \\
\hline FwpGAN-ISceI $(P m e I)$ & $\mathrm{f}$ & GGTTTAAACCTAGGGATAACAGGGTAATTCGCCCTTGCTCTAGATAAC & pCRpyrGAN \\
\hline RevpGAN-ISceI(PmeI) & $\mathrm{r}$ & GGTTTAAACCTAGGGATAACAGGGTAATAATTCGCCCTTGACTAGTGC & pCRpyrGAN \\
\hline GSP5 (AsiSI) & $\mathrm{f}$ & GCGATCGCACGCAAACCCATCGTTCC & gDNA QM6a \\
\hline GSP6 (AsiSI) & $\mathrm{r}$ & GCGATCGCGGTTCACCGCCTTATGTGAG & gDNA QM6a \\
\hline
\end{tabular}

Underlined sequences within the primers denote the I-SceI restriction site

identify this putative NLS sequence in the $T$. reesei Velvet protein, the putative NLS sequence identified in the Velvet protein of $A$. nidulans (Stinnett et al. 2007) was used for alignments.

\section{Construction of the glucoamylase expression cassette for targeted integration}

The plasmid pTrex6g-GA (Bower et al. 2012), which harboured the wild-type glucoamylase gene of $T$. reesei under control of the $c b h I$ promoter, was used to construct the glucoamylase expression cassette for integration at the I-SceI landing sites. To allow homologous integration of this reporter, the $c b h 2$ terminator region (Tcbh2) was cloned in pTrex6gGA. The Tcbh 2 was amplified by PCR using the primers GSP5 and GSP6 (Table 2) and the genomic DNA of the T. reesei QM6a WT strain. The PCR product was digested with $A s i \mathrm{SI}$ and cloned into the same restriction sites of pTrex6g-GA, to form plasmid pJP8. The 10-kb glucoamylase expression fragment was cut out from pJP8 with PsiI and used for transformation.

\section{In vivo analysis of I-SceI activity in $T$. reesei and fluorescent microscopy}

Purified T. reesei transformants that carried I-SceI restriction sites and the I-SceI expression cassette were point inoculated on TrMM with $2 \%$ of glucose or lactose as a carbon source with or without addition of uridine to the medium. The activity of I-SceI was monitored by formation of sectors without growth after incubating transformants for several days at $30^{\circ} \mathrm{C}$. Sector formation is likely to be the consequence of excision of the $p y r G$ marker and subsequent repair of the genomic DNA induced by the I-SceImediated DSB. Transformants that formed sectors on solid medium were selected for GFP expression under fluorescence microscopy. A total of $10^{7}$ conidia of sector-forming transformants were inoculated in 5 -mL germination medium containing $2 \%$ glucose/sophorose (30:1) for induction and supplemented with $0.003 \%$ yeast extract and $10 \mathrm{mM}$ uridine, and cultivated on cover slips for $28 \mathrm{~h}$ at $30{ }^{\circ} \mathrm{C}$. Samples were observed with Axioplan 2 fluorescence microscope (Zeiss, Sliedrecht, The Netherlands) equipped with a DKC-5000 digital camera (Sony) using different contrast or GFP settings. Images were captured and processed using Adobe Photoshop 6.0 (Adobe Systems Inc.).

\section{Quantification of the efficiency of $p y r G$ excision mediated by I-SceI expression}

To determine the frequency of the $\operatorname{pyr} G$ marker loss as a consequence of I-SceI expression, the strain JP7.7.12 bearing the I-SceI restriction sites and the I-SceI expressing cassette (pTTT-ISceI) was used. As a control, strain JP7.7_pTTT bearing the I-SceI restriction sites and the pTTT plasmid without I-SceI was included. About 100 spores of each strain harvested from minimal medium without uridine were grown separately under inducing conditions (TrMM with $2 \%$ lactose as carbon source) supplemented with uridine. Addition of uridine into the medium allowed for growth of all spores including those that will lose the pyrG marker under I-SceI expression conditions. To quantify the frequency of the $\operatorname{pyr} G$ loss events, single colonies from I-SceI-induced medium were transferred into minimal medium with and without uridine. The efficiency of the marker excision was determined as the ratio of the number of $p y r G$-negative colonies and the total number of colonies grown on the plate with uridine.

\section{Determination of glucoamylase activity and glucoamylase levels}

Purified transformants were grown in production medium for 5 days and glucoamylase was measured from the culture 
filtrate. The activity of glucoamylase was determined using Betamyl as a substrate (Megazyme International, Bray, Ireland). Ten microlitres of the culture samples containing glucoamylase were mixed with $90 \mu \mathrm{L}$ of Betamyl diluted in $50 \mathrm{mM}$ sodium acetate $\mathrm{pH}$ 4.8. The reaction is performed at $37{ }^{\circ} \mathrm{C}$ for $20 \mathrm{~min}$ and quenched with $50 \mu \mathrm{L}$ of $1 \mathrm{M}$ sodium carbonate $\mathrm{pH}$ 9. Activity was monitored by the release of $p$-nitrophenol measured colorimetrically at $405 \mathrm{~nm}$. For each transformant, the glucoamylase activity was measured in triplicate. Glucoamylase levels were detected using $10 \%$ sodium dodecyl sulfatepolyacrylamide gel electrophoresis (SDS-PAGE) performed under denaturing conditions according to Laemmli (1970). Gels were stained with Coomassie Brilliant Blue R-250 (Bio-Rad, Veenendaal, The Netherlands).

\section{Results}

\section{Design of a marker excision method to monitor I-SceI activity}

With the aim to generate I-SceI-mediated double-strand DNA breaks at a predetermined site in the $T$. reese $i$ genome for targeted integration, we first wished to make sure that the expression of the $S$. cerevisiae gene encoding I-SceI results in an active protein. To monitor the activity of the I-SceI meganuclease in $T$. reesei, a reporter construct that contained two I-SceI recognition sites flanking the A. nidulans pyrG selective marker was designed (Fig. 1). In addition, DNA with GFP direct repeats encoding an $\mathrm{N}$ terminally $(\triangle N G F P)$ and C-terminally $(G F P \triangle C)$ truncated, non-functional GFP were inserted around the I-SceI-pyrG-ISceI sequence to provide regions for homologous recombination. Transcription of the GFP $\triangle C$ part was regulated by the $c b h I$ promoter. The pCbh1-GFP $\triangle C$-I-SceI-pyrG-ISceI- $\triangle N G F P$ construct was subsequently cloned between $\sim 1.5 \mathrm{~kb} 5^{\prime}$ and $3^{\prime}$ regions of the $c b h 2$ gene $(0.6 \mathrm{~kb}$ in length) for targeted integration at the cbh2 locus. The rationale of the strategy is that active I-SceI expressed in a $T$. reesei strain containing this reporter construct would result in excision of the pyrG marker and repair of the double-strand break via the GFP repeats. A perfect repair would result in a uridine-auxotrophic strain expressing GFP (Fig. 1). It should be noted that the genome of $T$. reesei does not naturally contain a I-SceI restriction site (5'-TAGGGATAACAGGGTAAT-3').

\section{Construction of a $T$. reesei strain harbouring the I-SceI restriction sites at the $c b h 2$ locus}

The 7.5-kb reporter cassette as described above was linearized and introduced into a $T$. reesei strain deleted for $c b h I$.
Transformants were analysed by Southern blot for correct integration at the $c b h 2$ locus. Southern blot analysis revealed three transformants harbouring a single copy of the complete I-SceI cassette at the cbh2 locus (JP7.7, JP7.9 and JP7.12) (Fig. S2 in the Supplementary Material). Other transformants either had multiple copies of the cassette integrated (JP7.10, JP7.11, JP7.13, JP7.14 and JP7.15) or did not seem to insert the complete cassette into the genome (JP7.8) (Fig. S2 in the Supplementary Material). Transformant JP7.7 was used in subsequent experiments.

\section{I-SceI expression in $T$. reesei induces DSB at the targeted chromosomal locus}

A codon-optimized version of I-SceI with or without an additional NLS sequence from the $T$. reesei vell gene was introduced on a replicative plasmid into JP7.7 strain bearing the I-SceI landing sites. Expression of ISceI nuclease was controlled by the cbhl promoter, which is repressed by glucose and strongly induced by lactose or sophorose (Ivanova et al. 2013; Xu et al. 2014). Transformants were grown either on glucose- or lactose/glucose-containing medium. As shown in Fig. 2, induction by lactose in the strains expressing I-SceI and containing the I-SceI reporter cassette resulted in sectored colonies. This sectoring could be interpreted as I-SceI activity generating a double-strand break, which was repaired via recombination between the GFP direct repeats leading to loss of the pyrG marker. As a consequence of efficient recombination in all nuclei in some part of the colony, this part of the colony no longer grew on plates without uridine giving rise to a nongrowing sector. As shown in Fig. 2, sectors were present when lactose was used as a carbon source, although we occasionally (less than $10 \%$ of the glucose/lactose plates) also observed sectors on glucose plates indicating that some I-SceI might be expressed (not shown). No sectors were observed in the control strains (JP7.7) or JP7.7 containing the empty vector (JP7.7+ pTTT), indicating the SceI expression is required for looping out the pyrG marker. Similar sectoring on lactose was observed for transformants expressing I-SceI with NLS $_{\text {vel1 }}$ but never for the control strains (Fig. 2 and data not shown). When uridine was added to the plates, non-growing sectors were absent indicating that they were caused by loss of the pyrG marker (Fig. 2). These results strongly suggest that the $S$. cerevisiae ISceI was expressed and active in $T$. reesei.

Functionality of I-SceI was further confirmed by fluorescent microscopy in liquid cultures. Hyphae grown in the presence of sophorose showed green fluorescence indicating reconstitution of a functional GFP as a consequence of the pyrG excision followed by a 


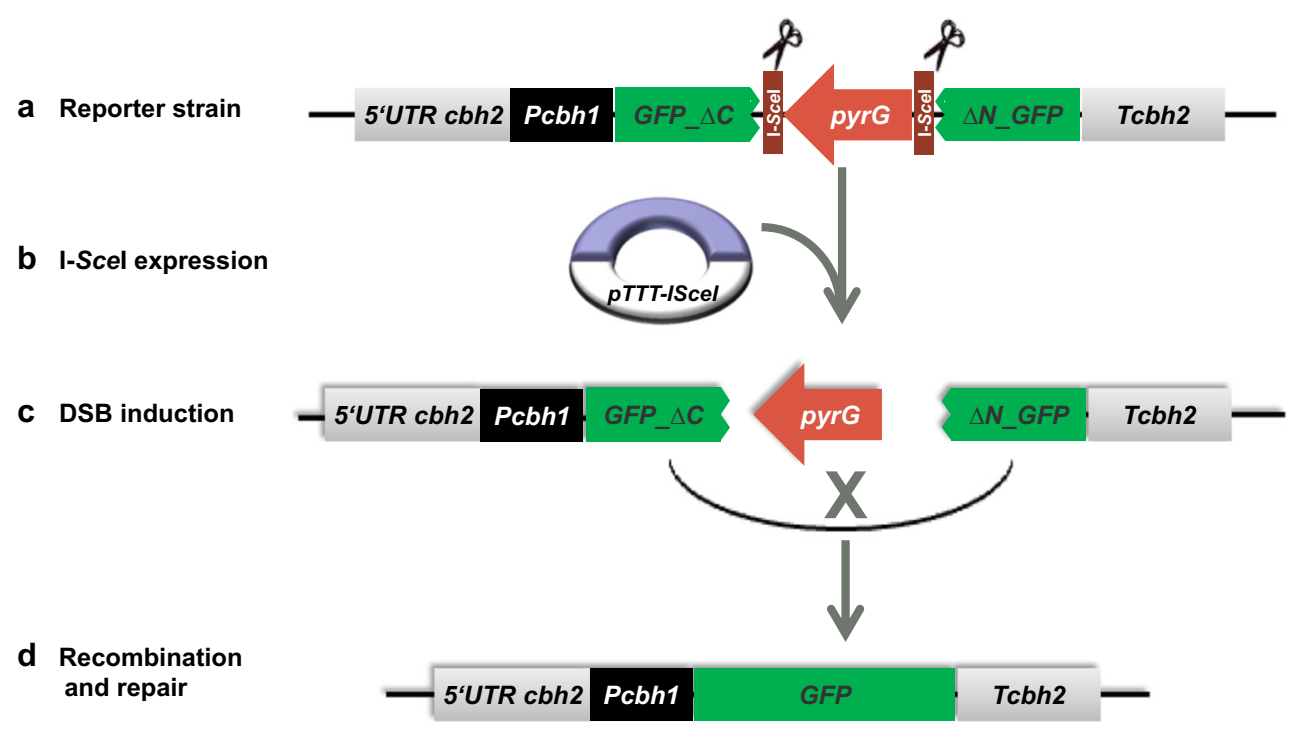

Fig. 1 Schematic representation of the strategy to test the doublestrand break (DSB) activity of I-SceI expressed in T. reesei. The reporter cassette (pBJP6) with I-SceI restriction sites inserted at the $c b h 2$ locus of a $T$. reesei uridine auxotrophic strain results in a uridine prototrophic strain containing two I-SceI sites surrounding the pyrG cassette $(a)$. Heterologous expression of I-SceI is expected to generate two DSBs and the loss of the $p y r G$ selection marker between the I-SceI sites (b). The DSB can be repaired by

recombination event. As GFP was observed only in strains expressing I-SceI, this further indicates that ISceI was actively expressed (Fig. 3).

The frequency of the I-SceI-mediated excision was determined by comparing the frequency of losing the $p y r G$ marker in the I-SceI-expressing and I-SceI-non-expressing strains (strains JP7.7.12 and JP7.7_pTTT, respectively). Therefore, about 100 spores of each strain were plated on lactosecontaining induction medium supplemented with uridine. After growth for 3 days, each colony formed was further scored for its growth in the absence of uridine. Sixty-six percent of the colonies of the JP7.7.12 strain bearing the I-SceI restriction sites and containing the I-SceI expression cassette (pTTT-ISceI) were found to be auxotrophic for uridine, whereas only $1.3 \%$ of the colonies of JP7.7_pTTT strain bearing the empty pTTT plasmid were uridine-dependent. Thus, a high frequency of $p y r G$ excision mediated by in vivo-expressed I-SceI nuclease was observed during a plate growth test.

\section{I-SceI-mediated DSB increases transformation efficiency and transformant stability}

To determine whether a DSB mediated by I-SceI can increase transformation and recombination efficiency in T. reesei, the aforementioned reporter strain JP7.7.12 was transformed with a linear fragment comprising a glucoamylase expression cassette (Fig. 4). This cassette homologous recombination between $G F P \triangle C$ and $\triangle N G F P$ adjacent to the breaks $(c)$. After homologous recombination via the GFP direct repeats, this will result in a uridine auxotrophic strain (loss of the $\operatorname{pyr} G$ gene) and reconstitution of a functional GFP when correctly recombined via the direct repeat sequences $(d)$. The resulting strain can be either screened for uridine auxotrophy or/and for GFP expression to monitor I-SceI activity

contains the alS selection marker which renders transformations to become resistant against chlorimuron ethyl (Bower et al. 2012). Chlorimuron ethyl-resistant transformants were selected either on glucose (I-SceI-repressed conditions) or lactose- and glucose-containing medium (I-Scel-inducing condition). As a control, we also transformed a strain carrying only the I-SceI restriction sites (JP7.7) with the same DNA fragment. As shown in Fig. 5 and Table 3, I-SceI induction increased the number of chlorimuron ethyl-resistant transformants more than threefold and sixfold as compared to repressed conditions and to the control, respectively.

We further analysed the stability of the transformants obtained in each group of transformation, as by subsequent purification on MM-chlorimuron ethyl plates. Ninety percent of the transformants obtained after the induction of I-SceI were stable against 44 and $58 \%$ of colonies grown under repressed conditions and for the control strain, respectively (Table 3). This indicates that heterologous expression of the I-SceI nuclease increased transformation efficacy and transformant stability. Instability of transformants usually results from a lack of integration of a given DNA cassette into the genome and has been previously described to be a major bottleneck for $T$. reesei transformation (Jørgensen et al. 2014).

Targeted integration of the glucoamylase expression cassette at the predetermined genomic locus would result in 


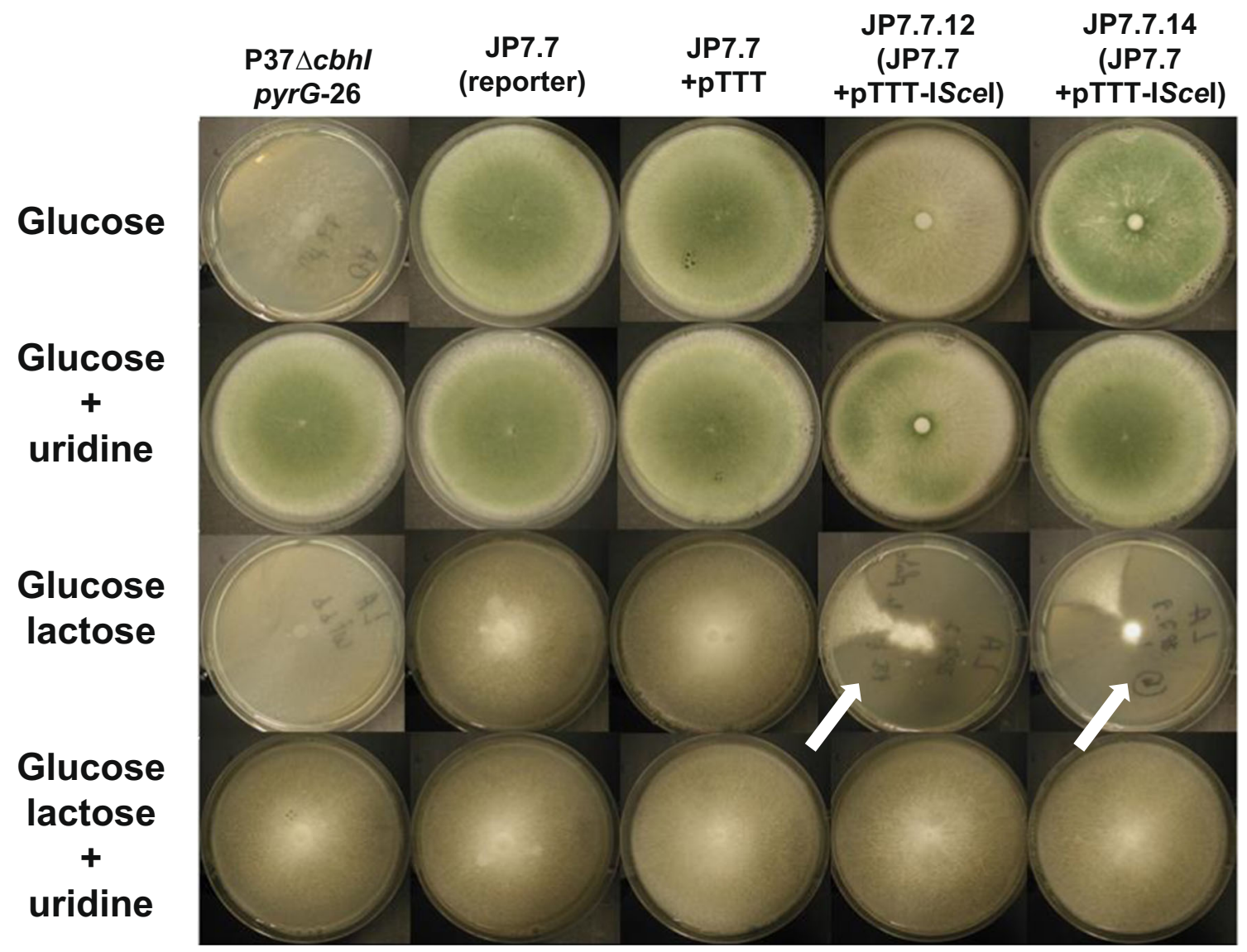

Fig. 2 Loss of the $p y r G$ cassette is mediated via I-SceI meganucleaseinduced loop-out recombination control strain (P37 $\Delta$ cbhIpyrG-26), and strains harbouring the I-SceI restriction site-containing construct (JP7.7 transformants) were transformed with either the control plasmid (pTTT) or the I-SceI expression plasmid (pTTT-ISceI). Spores of the strains were point-inoculated in the centre of a 9-cm Petri dish containing minimal medium and containing either $50 \mathrm{mM}$ glucose (glucose) or a mix of $50 \mathrm{mM}$ glucose and $50 \mathrm{mM}$ lactose (glucose and lactose) either with or without the addition of uridine. Pictures of the colonies were taken after
4 days of growth at $30{ }^{\circ} \mathrm{C}$. Induction of I-SceI expression by lactose in JP7.7 transformants containing the pTTT-ISceI vector results in sectored colonies. These sectors are regions of no growth expected to be caused by loop out of the $p y r G$ marker and the subsequent inability of the strain to grow on the medium without uridine. Sectoring is dependent on the expression of I-SceI via lactose induction and no longer visible if uridine is supplemented. Arrows indicate regions with no growth. As expected, the parental strain does not grow on media without uridine acquisition of chlorimuron ethyl resistance $\left(a l S^{+}\right.$phenotype), uridine auxotrophy, caused by a loss of the $\operatorname{pyr} G$ marker, and the presence of the glucoamylase expression cassette (Fig. 4). To determine whether the I-SceI-mediated targeted integration correlated with the expression of the introduced glucoamylase cassette, we assayed $\sim 40$ stable transformants from each transformation group for glucoamylase activity and screened the glucoamylasepositive transformants for their $p y r G$ phenotype. As shown in Table 3, about two thirds of the glucoamylase-positive transformants (15 of 22) obtained upon I-SceI induction became uridine-auxotrophic, indicating that the construct was integrated at the intended locus via a doublecrossover event, thereby removing the $\operatorname{pyr} G$ marker. Frequencies of homologous recombination were $\sim 46$ and $\sim 16 \%$ under I-SceI repression and in the control strain, respectively. These results show that expression of I-SceI increased both the transformation efficiency and the frequency of targeted integration in $T$. reesei. Leaky expression from the cbhI promoter in glucose medium could explain the increased number of targeted integration transformants under repressed conditions, as compared to the control transformants. Overall analysis showed that $\sim 93 \%(14 / 15)$ uridine-requiring strains expressed glucoamylase when I-SceI was induced, thereby confirming strong correlation between a $p y r G$-negative phenotype and glucoamylase expression. We further analysed genomic DNA from selected transformants for the integration pattern of the glucoamylase cassette by Southern blot. The uridineauxotrophic transformants expressing glucoamylase contained the complete glucoamylase cassette integrated in a single copy at the predetermined cbh2 locus (Fig. 6a, b). $\mathrm{PyrG}^{+}$transformants not showing glucoamylase activity very likely did not or only partially integrated the 


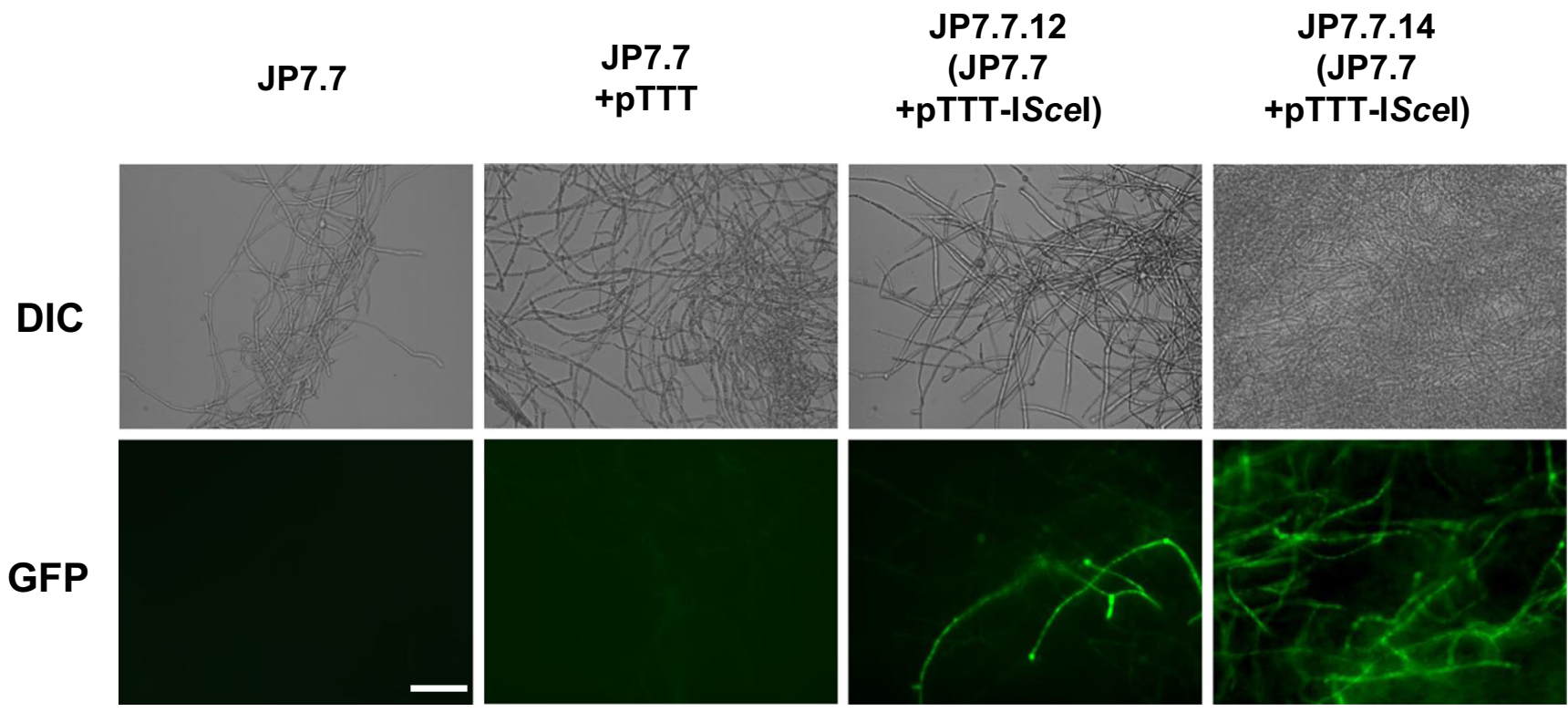

Fig. 3 Visualization of GFP fluorescence after I-SceI meganucleasemediated loop-out recombination. Induction of I-SceI expression by sophorose results in excision of the $p y r G$ marker and reconstitution of a functional GFP, indicated by the green fluorescent hyphae. Strains were grown on TrMM containing $2 \%$ glucose/sophorose (30:1) and uridine to

glucoamylase cassette. $P y r G^{+}$transformants displaying glucoamylase activity resulted probably from a partial induce I-SceI expression and allow the loss of the $p y r G$ marker, respectively. Strain JP7.7, harbouring I-SceI restriction sites but not expressing the I-SceI gene, was included in the analysis. Lack of GFP expression indicates that the loop-out event to restore GFP functionality requires I-SceI. Bars, $10 \mu \mathrm{m}$

integration of the cassette with the intact glucoamylase gene into the genome (Fig. 6a, b; PCR, data not shown).

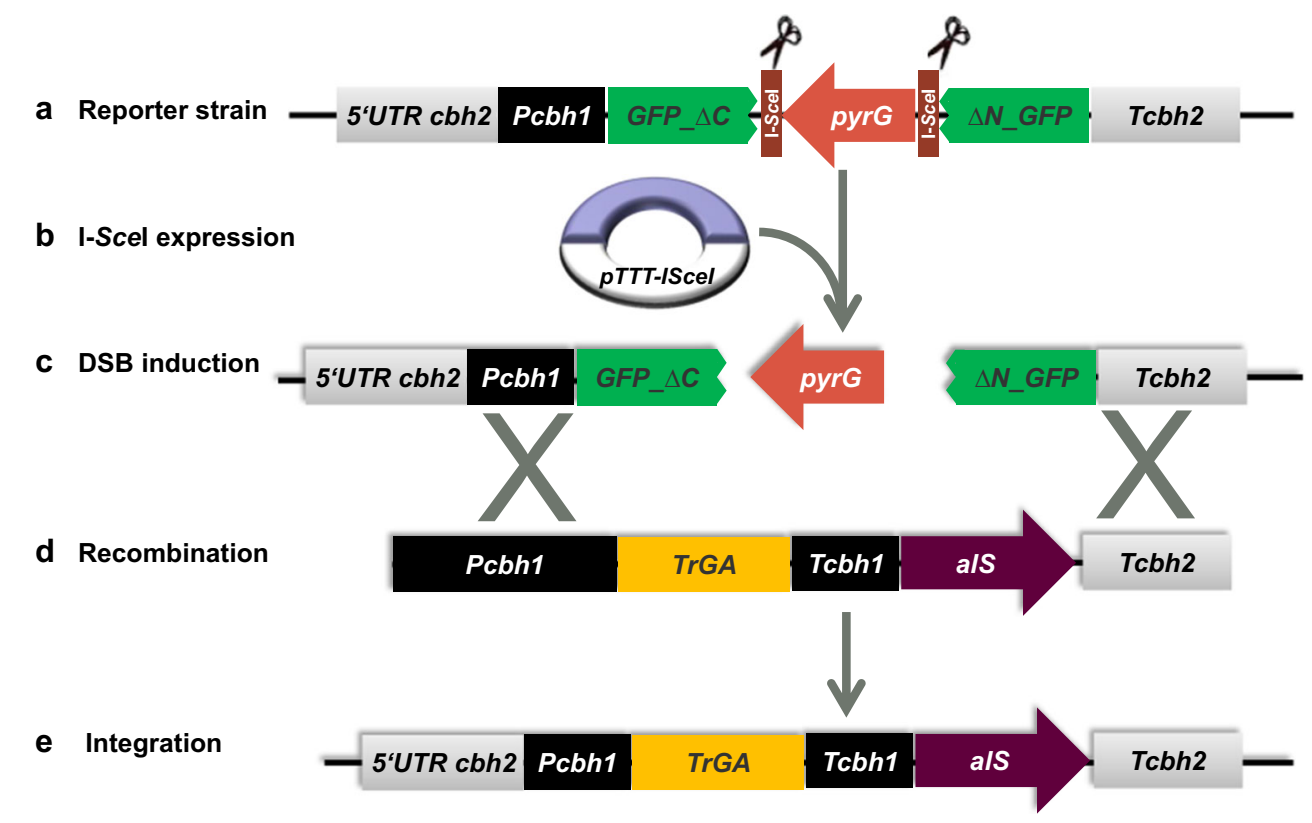

Fig. 4 Schematic representation of targeted integration of the glucoamylase expression cassette via I-SceI-mediated homologous recombination. (a) As a starting strain, the same reporter strain (JP7.7) was used as before. This strain contains the cassette with the I-SceI restriction sites inserted at the cbh2 locus of $T$. reesei. (b) Transformation of JP7.7 with the vector to express the I-SceI gene (pTTT-ISceI). This strain can be propagated on glucose medium without uridine to keep selection pressure on maintaining the $p y r G$ cassette. ( $c$ and $d$ ) Transformation of the glucoamylase-expressing cassette (pJP8) and simultaneous induction of I-SceI expression to create a DSB. I-SceI was induced by plating out protoplasts on lactosecontaining transformation plates. The DSB can be repaired by homologous recombination with the glucoamylase-expressing cassette (pJP8), which has homology regions to the locus containing the I-SceI sites. (e) Targeted integration of the glucoamylase cassette at the I-SceI landing site would generate a strain that is resistant to chlorimuron ethyl $\left(a l S^{+}\right)$and uridine auxotrophic $\left(\right.$pyr $\left.G^{-}\right)$and is expected to contain the glucoamylase gene under control of the $c b h I$ promoter 


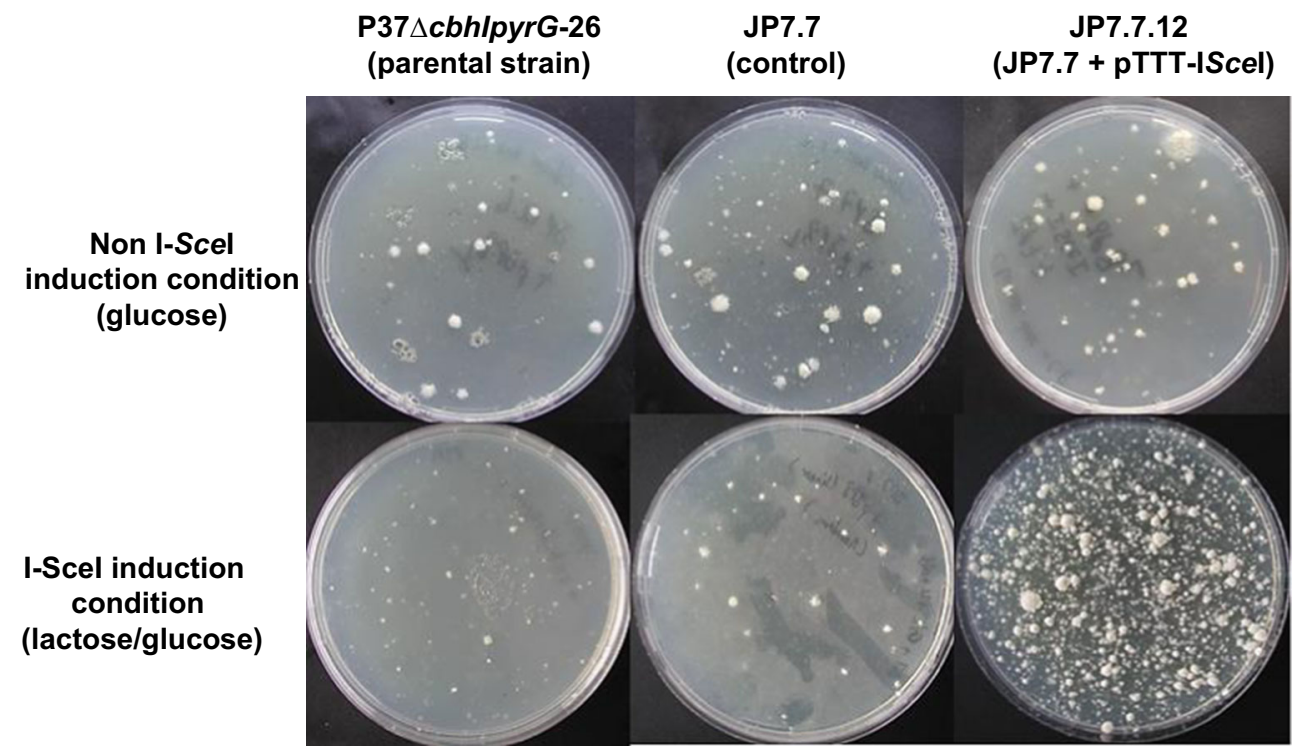

Fig. 5 Transformation under I-SceI-inducing and non-inducing conditions. The glucoamylase-expressing cassette (pJP8) with homologous regions to the locus containing the I-SceI restriction sites was transformed into the parental strain (P37), JP7.7 (containing the ISceI restriction site construct) and JP7.7.12 (containing the I-SceI restriction site construct and expressing I-SceI), respectively, by PEG transformation. The protoplasts were plated out on glucose- or lactose/ glucose-based transformation medium supplemented with chlorimuron ethyl and uridine. Transformants were photographed after 3 days of incubation

\section{Uniform expression of the reporter glucoamylase gene after targeted integration at the engineered genomic locus}

To compare the variability in glucoamylase (GA) expression among transformants with targeted integration of the GA cassette ( $p y r G^{-}$transformants) versus random integrated transformants ( $p y r G^{+}$transformants), strains were grown in sophorose-containing medium to induce GA, and culture samples were assayed for glucoamylase activity. As shown in Fig. 7, pyr $G^{-}$transformants obtained displayed a more consistent pattern of glucoamylase activity as compared to the expression profile of $\mathrm{pyr}^{+}$transformants. Nearly $80 \%$ of transformant GA activity varied within a range of $15-20 \%$, which could be explained by a single-copy integration of the GA fragment at the engineered locus. The variation observed resulted from fermentation conditions rather than differences in the genetic background of the transformants. In contrast, a random integration of the expression cassette gave rise to significant variation in GA levels among transformants (Fig. 7). On average, the transformant obtained by random integration

Table 3 The effect of I-SceI induction on number and stability of transformants

\begin{tabular}{|c|c|c|c|c|c|c|}
\hline Strain & C source & $\begin{array}{l}\text { \# of primary } \\
\text { transformants }^{\mathrm{a}}\end{array}$ & $\begin{array}{l}\% \text { stable } \\
\text { transformants }^{\mathrm{b}}\end{array}$ & $\begin{array}{l}\% \text { Gla-positive } \\
\text { transformants }^{\mathrm{c}}\end{array}$ & $\begin{array}{l}\% \text { Gla-positive pyrG } \\
\text { transformants }\end{array}$ & $\begin{array}{l}\text { Homologous recombination } \\
\text { efficiency }\end{array}$ \\
\hline JP7.7 (Control) & Lactose & 32 & $17 / 32(53 \%)$ & Not tested & Not tested & Not tested \\
\hline JP7.7 (Control) & Glucose & 36 & $21 / 36(58 \%)$ & $12 / 40(30 \%)$ & $2 / 12(16 \%)$ & $2 / 12(16 \%)$ \\
\hline JP7.7.12 (pTTT-ISceI) & Lactose & $>226$ & $45 / 50(90 \%)$ & $22 / 40(55 \%)$ & $15 / 22(68 \%)$ & $15 / 22(68 \%)$ \\
\hline JP7.7.12 (pTTT-ISceI) & Glucose & 70 & $22 / 50(44 \%)$ & $15 / 40(37 \%)$ & $7 / 15(46 \%)$ & $7 / 15(46 \%)$ \\
\hline
\end{tabular}

${ }^{a}$ Number of primary transformants on transformation plates (TrMMsorb + uridine + chlorimuron ethyl (Als substrate)) containing $1 \%$ of lactose and $1 \%$ glucose referred to as induced and non-induced expression of I-SceI, respectively. Control transformations have been carried out using the transformation medium using the parental strain JP7.7.

${ }^{\mathrm{b}}$ Primary transformants were purified on TrMM + uridine + chlorimuron ethyl. The stability of the transformants is defined by their ability to grow in selective TrMM after double purification. Stable transformants grow well on these selective plates. Abortive transformants (not stable) do not grow

${ }^{\mathrm{c}}$ Stable transformants were tested for glucoamylase activity in a microtitre-based growth/activity assay. Transformants showing Gla activity above the background were considered as Gla-positive transformants

${ }^{\mathrm{d}}$ Gla-positive transformants were tested for the $p y r G$ phenotype by inoculating spores of each transformant on TrMM with uridine or TrMM without uridine. Strains were considered $\operatorname{pyr} G$ minus (uridine auxotroph) when growing on TrMM + uridine, but not growing om TrMM without uridine

${ }^{\mathrm{e}} \mathrm{GlaA}$ expressing and $\operatorname{pyr} G$ minus strain were analysed by Southern blot and confirmed an integration pattern indicative of homologous integration of the glaA expression cassette at the I-SceI landing site 
a

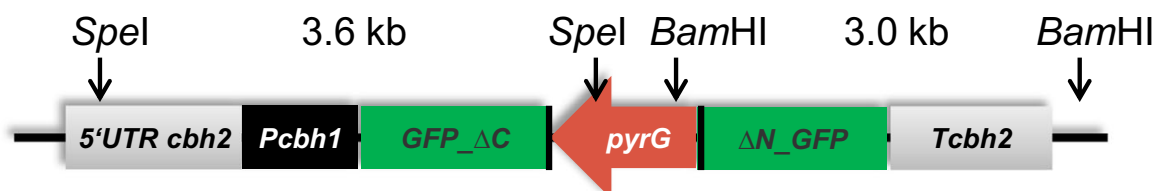

probe A

probe B

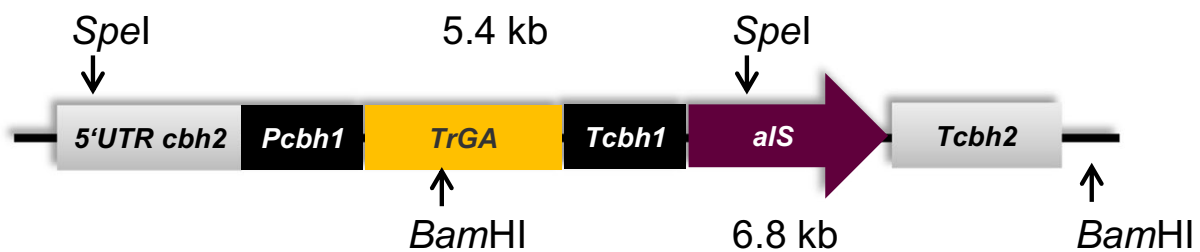

b

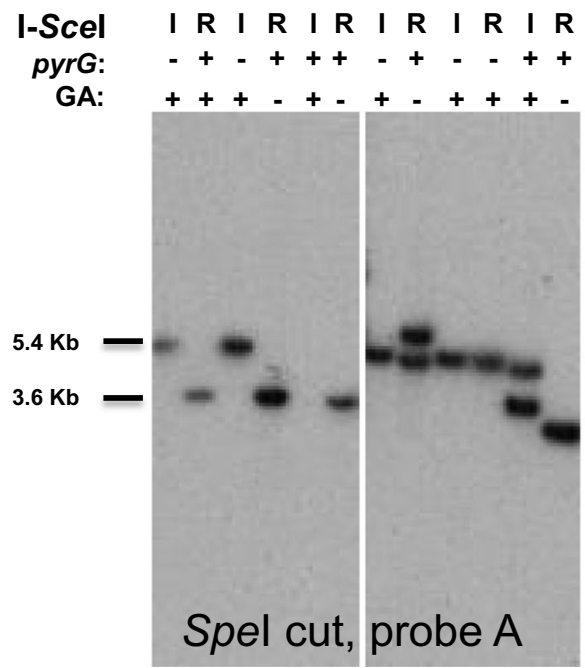

Fig. 6 Southern blot analysis of selected transformants after transformation with the glucoamylase expression cassette. a Diagram of pJP8 transformants to analyse integration of the glucoamylase cassette at the 5' flank and 3' flank of the cbh2 locus by using SpeI and BamHI as restriction enzymes and $P c b h I$ and Tcbh2 as probes, respectively. The expected band size is $5.4 \mathrm{~kb}$ for integration of the glucoamylase cassette using the PcbhI probe and $6.8 \mathrm{~kb}$ for integration using the Tcbh 2 probe. A non-homologous integration of the glucoamylase cassette will not alter the cbh2 locus and a hybridizing DNA fragment

displayed lower glucoamylase activity than the transformant obtained by targeted integration and could be due to the targeting of the expression cassette to the $c b h 2$ locus, which is likely to be an actively transcribed region with a positive effect on glucoamylase production which is driven by the $c b h 1$ promoter.

\section{Discussion}

Rare-cutting, double-stranded DNA endonucleases, called meganucleases, have emerged as a powerful tool for

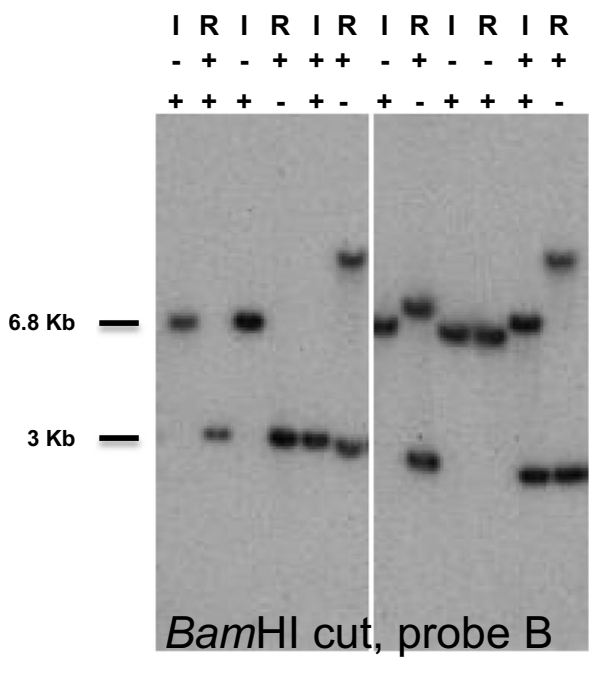

of 3.6 and $3 \mathrm{~kb}$ are expected with the $P c b h I$ probe and the $c b h 2$ probe, respectively. b Southern blot results of pJP8 transformants to analyse the integration of the glucoamylase cassette at the $5^{\prime}$ flank (left blot) and $3^{\prime}$ flank (right blot) of the cbh2. The two blots shown for analysis of the $5^{\prime}$ flank integration or $3^{\prime}$ flank integration are from a single Southern blot. Some lanes of the blot have been removed to exclude unrestricted genomic DNA samples. I I-SceI is induced, $R \mathrm{I}-S c e \mathrm{I}$ is repressed, + indicate presence of $\operatorname{pyr} G$ marker or glucoamylase expression, indicate absence of the pyr $G$ marker or non-expression of glucoamylase

genome manipulation. Several studies have demonstrated that expression of such endonucleases in prokaryotic and eukaryotic cells stimulated homologous recombination between a given repair template and a genome locus containing an endonuclease recognition site (Choulika et al. 1995; Glover and Horn 2009; Meddows et al. 2005; Rouet et al. 1994). The aim of the current study was to increase efficiency of the gene-targeted integration at a predetermined locus in the $T$. reesei genome to ensure high expression levels and limited variability of protein expression. To this end, the $S$. cerevisiae endonuclease ISceI was expressed in T. reesei and I-SceI recognition sites flanking a reporter construct were introduced into the $c b h 2$ 
Variability of GA activity between transformants

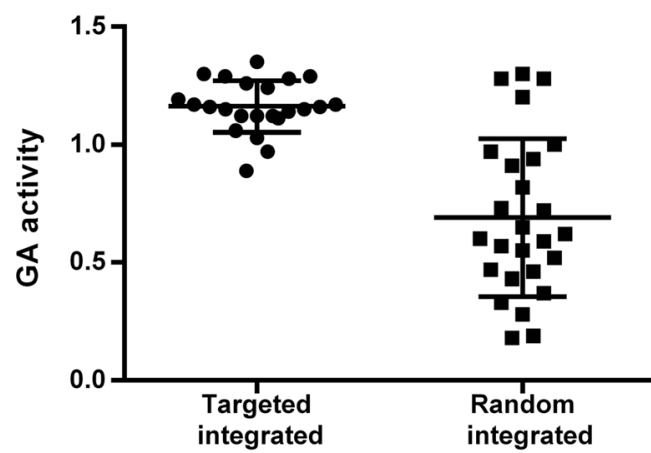

Transformants

Fig. 7 Glucoamylase activity in culture medium of transformants obtained after targeted or random integration. Targeted integrated transformants displayed high homogeneity of glucoamylase activity compared to transformants with random integration of the glucoamylase cassette. The analysed transformants were tested based on their auxotrophy to uridine. The targeted $(n=23)$ and random $(n=26)$ integrated transformants are $p y r G^{-}$and $p y r G^{+}$ phenotype, respectively. The $p y r G^{-}$transformants were further characterized for correct targeting of the glucoamylase cassette at the $c b h 2$ locus. Plots were created using GraphPad Prism 6 (column scatter graph). The horizontal bars represent the mean values with standard deviations

locus of $T$. reesei. We demonstrated that expression of ISceI resulted in endonuclease activity. The I-SceI protein appeared to be imported into the $T$. reesei nucleus via the intrinsic NLS signal, as we did not detect obvious differences in efficiency of marker excision between the original I-SceI protein and a I-SceI variant fused to a nuclear targeting signal of the $T$. reesei Vell protein. We analysed the impact of the I-SceI-mediated DSBs on transformation frequency, transformant stability and efficiency of homologous recombination.

Our data indicate that DSB mediated by I-SceI in T. reesei improves the efficiency of transformation and the stability of the transformants and promotes gene targeting at a defined locus. At least sixfold more transformants were obtained when I-SceI was induced, and, more importantly, $90 \%$ of them were found to be stable. Genetic instability is a major bottleneck in $T$. reesei transformation and usually is a consequence of a lack of integration of a transformed DNA cassette into the genome or due to a tandem integration of multiple copies, which could be excised through a loop-out event (Aw and Polizzi 2013; Jørgensen et al. 2014; Le Dall et al. 1994; Lee and Da Silva 1997; Ohi et al. 1998). This is the first study that addresses issues related to the stability of transformants mediated by I-SceI in filamentous fungi and particularly in the industrial fungus $T$. reesei. Furthermore, when I-SceI was expressed in the cells, the frequency of homologous recombination increased up to $68 \%$.
A recent study of gene targeting based on I-SceI-induced DSBs in P. oryzae (Magnaporthe oryzae) reported that in this fungus, I-SceI expression increases targeted integration up to about $40 \%$, which is comparable to our findings (Arazoe et al. 2014). Targeted integration can be also improved in filamentous fungi by deletion of the genes involved in non-homologous end joined (NHEJ) recombination (Ninomiya et al. 2004; Krappmann et al. 2006; Meyer et al. 2007; Guangtao et al. 2009; Steiger et al. 2011). However, inactivation of the NHEJ genes usually increases strain sensitivity towards chemicals and physical DNA-damaging agents and mostly reduces efficiency of transformation as compared to a strain with intact NHEJ genes (Zhang et al. 2011). In addition, targeted integration in strains deficient of NHEJ proteins can be highly locus-dependent and may vary from 33 to $100 \%$ depending on the insertion site (Jørgensen et al. 2014; Schuster et al. 2012). Our study shows that using the I-SceI endonuclease, we can improve targeted integration events in a $T$. reesei strain with intact NHEJ genes. Moreover, the targeted integration transformants could be specifically selected by adding 5-fluoroorotic acid (5'FOA) in the transformation medium. About $90 \%$ of the $p y r G$ transformants expressed glucoamylase, indicating that the DNA cassette was integrated at the intended locus. Moreover, Southern blot analysis confirmed the presence of a single copy of the expression fragment in these transformants. Importantly, we found that glucoamylase production levels were much more consistent among the transformants that harboured the expression cassette at the targeted locus as compared to transformants with a random integration pattern. Homogeneity in protein expression in the population of transformants is always a great challenge in the field of fungal biotechnology because of the low efficiency of targeted integration events (Aw and Polizzi 2013). Thus, our technique may be employed for high-throughput screening of enzyme libraries or for construction of production strains with predetermined integration sites, which is especially relevant in industrial biotechnology.

Acknowledgments We thank Prof. Dr. Paul Hooykaas and Dr. Jaap Visser for the helpful discussions. We thank Dr. F. Klis for carefully reading the manuscript. This project was carried out within the research programme of the Kluyver Centre for Genomics of Industrial Fermentation, which is part of The Netherlands Genomics Initiative/Netherlands Organization for Scientific Research.

Conflict of interest The authors declare that they have no competing interests.

Compliance with ethical standards This article does not contain any studies with human participants or animals performed by any of the authors. 
Open Access This article is distributed under the terms of the Creative Commons Attribution 4.0 International License (http:// creativecommons.org/licenses/by/4.0/), which permits unrestricted use, distribution, and reproduction in any medium, provided you give appropriate credit to the original author(s) and the source, provide a link to the Creative Commons license, and indicate if changes were made.

\section{References}

Adrio JL, Demain AL (2014) Microbial enzymes: tools for biotechnological processes. Biomolecules 4:117-139. doi:10.3390/biom4010117

Aehle, W, Bott, RR, Nikolaev, I, Scheffers, M, van Solingen, P, Vroemen, C (2011) Glucoamylase variants with altered properties. Patent US20110020899. Accessed 27 January 2011

Anderson LN, Culley DE, Hofstad BA, Chauvigné-Hines LM, Zink EM, Purvine SO, Smith RD, Callister SJ, Magnuson JM, Wright AT (2013) Activity-based protein profiling of secreted cellulolytic enzyme activity dynamics in Trichoderma reesei QM6a, NG14, and RUT-C30. Mol BioSyst 9:2992-3000. doi:10.1039/c3mb70333a

Arazoe T, Younomaru T, Ohsato S, Kimura M, Arie T, Kuwata S (2014) Site-specific DNA double-strand break generated by I-SceI endonuclease enhances ectopic homologous recombination in Pyricularia oryzae. FEMS Microbiol Lett 352:221-229. doi:10.1111/15746968.12396

Aw R, Polizzi KM (2013) Can too many copies spoil the broth? Microb Cell Factories 12:128. doi:10.1186/1475-2859-12-128

Bollag RJ, Waldman AS, Liskay RM (1989) Homologous recombination in mammalian cells. Annu Rev Genet 23:199-225. doi:10.1146/ annurev.ge.23.120189.001215

Bower BS, Dunn-Coleman N, Leiva N (2012). Acetolactate synthase (ALS) selectable marker from Trichoderma reesei. Patents US8138321. Accessed 20 March 2012

Cahill D, Connor B, Carney JP (2006) Mechanisms of eukaryotic DNA double strand break repair. Front Biosci 11:1958-1976

Catalano V, Vergara M, Hauzenberger JR, Seiboth B, Sarrocco S, Vannacci G, Kubicek CP, Seidl-Seiboth V (2011) Use of a nonhomologous end-joining-deficient strain (delta- $k u 70$ ) of the biocontrol fungus Trichoderma virens to investigate the function of the laccase gene $l c c 1$ in sclerotia degradation. Curr Genet 57:13-23. doi:10.1007/s00294-010-0322-2

Choulika A, Perrin A, Dujon B, Nicolas JF (1995) Induction of homologous recombination in mammalian chromosomes by using the ISceI system of Saccharomyces cerevisiae. Mol Cell Biol 15:19681973

Fairhead C, Dujon B (1993) Consequences of unique double-stranded breaks in yeast chromosomes: death or homozygosis. Mol Gen Genet 240:170-178

Glover L, Horn D (2009) Site-specific DNA double-strand breaks greatly increase stable transformation efficiency in Trypanosoma brucei. Mol Biochem Parasitol 166:194-197

Guangtao Z, Hartl L, Schuster A, Polak S, Schmoll M, Wang T, Seidl V, Seiboth B (2009) Gene targeting in a nonhomologous end joining deficient Hypocrea jecorina. J Biotechnol 139:146-151. doi:10. 1016/j.jbiotec.2008.10.007

Ivanova C, Baath JA, Seiboth B, Kubicek CP (2013) Systems analysis of lactose metabolism in Trichoderma reesei identifies a lactose permease that is essential for cellulase induction. PLoS ONE 8:e62631. doi:10.1371/journal.pone.0062631

Jørgensen MS, Skovlund DA, Johannesen PF, Mortensen UH (2014) A novel platform for heterologous gene expression in Trichoderma reesei (teleomorph Hypocrea jecorina). Microb Cell Factories 13: 33. doi:10.1186/1475-2859-13-33
Krappmann S, Sasse C, Braus GH (2006) Gene targeting in Aspergillus fumigatus by homologous recombination is facilitated in a nonhomologous end-joining-deficient genetic background. Eukaryot Cell 5:212-215. doi:10.1128/EC.5.1.212-215.2006

Kuijpers NG, Chroumpi S, Vos T, Solis-Escalante D, Bosman L, Pronk JT, Daran JM, Daran-Lapujade P (2013) One-step assembly and targeted integration of multigene constructs assisted by the I-SceI meganuclease in Saccharomyces cerevisiae. FEMS Yeast Res 13: 769-781. doi:10.1111/1567-1364.12087

Laemmli UK (1970) Cleavage of structural proteins during the assembly of the head of bacteriophage T4. Nature 227:680-685

Le Dall MT, Nicaud JM, Gaillardin C (1994) Multiple-copy integration in the yeast Yarrowia lipolytica. Curr Genet 26:38-44

Lee FW, Da Silva NA (1997) Improved efficiency and stability of multiple cloned gene insertions at the $\Delta$ sequences of Saccharomyces cerevisiae. Appl Microbiol Biotechnol 48:339-345

Meddows TR, Savory AP, Grove JI, Moore T, Lloyd RG (2005) RecN protein and transcription factor DksA combine to promote faithful recombinational repair of DNA double-strand breaks. Mol Microbiol 57:97-110. doi:10.1111/j.1365-2958.2005.04677.x

Meyer V, Arentshorst M, El-Ghezal A, Drews AC, Kooistra R, van den Hondel CA, Ram AF (2007) Highly efficient gene targeting in the Aspergillus niger kusA mutant. J Biotechnol 128:770-775. doi:10. 1016/j.jbiotec.2006.12.021

Meyer V, Ram AF, Punt PJ (2010) Genetics, genetic manipulation, and approaches to strain improvement of filamentous fungi. In: Demain AL, Davis J (eds) Manual of industrial microbiology and biotechnology, 3rd edn. John Wiley and Sons Inc, NewYork, pp 318-329

Monteilhet C, Perrin A, Thierry A, Colleaux L, Dujon B (1990) Purification and characterization of the in vitro activity of I-SceI, a novel and highly specific endonuclease encoded by a group I intron. Nucleic Acids Res 18:1407-1413

Ninomiya Y, Suzuki K, Ishii C, Inoue H (2004) Highly efficient gene replacements in Neurospora strains deficient for nonhomologous end-joining. Proc Natl Acad Sci U S A 101(33):12248-12253

Ohi H, Okazaki N, Uno S, Miura M, Hiramatsu R (1998) Chromosomal DNA patterns and gene stability of Pichia pastoris. Yeast 14:895903. doi:10.1002/(SICI)1097-0061(199807)14:10<895::AIDYEA288>3.0.CO;2-9

Penttilä M, Nevalainen H, Rättö M, Salminen E, Knowles J (1987) A versatile transformation system for the cellulolytic filamentous fungus Trichoderma reesei. Gene 61:155-164

Plessis A, Perrin A, Haber JE, Dujon B (1992) Site-specific recombination determined by I-SceI, a mitochondrial group I intron-encoded endonuclease expressed in the yeast nucleus. Genetics 130:451-460

Puchta H, Dujon B, Hohn B (1993) Homologous recombination in plant cells is enhanced by in vivo induction of double strand breaks into DNA by a site-specific endonuclease. Nucleic Acids Res 21:5034 5040

Rouet P, Smih F, Jasin M (1994) Introduction of double-strand breaks into the genome of mouse cells by expression of a rare-cutting endonuclease. Mol Cell Biol 14:8096-8106

Schuster A, Schmoll M (2010) Biology and biotechnology of Trichoderma. Appl Microbiol Biotechnol 87:787-799. doi:10. 1007/s00253-010-2632-1

Schuster A, Bruno KS, Collett JR, Baker SE, Seiboth B, Kubicek CP, Schmoll M (2012) A versatile toolkit for high throughput functional genomics with Trichoderma reesei. Biotechnol Biofuels 5:1. doi:10. 1186/1754-6834-5-1

Steiger MG, Vitikainen M, Uskonen P, Brunner K, Adam G, Pakula T, Penttilä M, Saloheimo M, Mach RL, Mach-Aigner AR (2011) Transformation system for Hypocrea jecorina (Trichoderma reesei) that favors homologous integration and employs reusable bidirectionally selectable markers. Appl Environ Microbiol 77:114-121. doi:10.1128/AEM.02100-10 
Stinnett SM, Espeso EA, Cobeno L, Araujo-Bazan L, Calvo AM (2007) Aspergillus nidulans VeA subcellular localization is dependent on the importin $\alpha$ carrier and on light. Mol Microbiol 63:242-255

Szostak JW, Orr-Weaver TL, Rothstein RJ, Stahl FW (1983) The doublestrand-break repair model for recombination. Cell 33:25-35

Turner NJ (2009) Directed evolution drives the next generation of biocatalysts. Nat Chem Biol 5:567-573. doi:10.1038/nchembio.203
Xu J, Zhao G, Kou Y, Zhang W, Zhou Q, Chen G, Liu W (2014) Intracellular $\beta$-glucosidases CEL1a and CEL1b are essential for cellulase induction on lactose in Trichoderma reesei. Eukaryot Cell 13:1001-1013. doi:10.1128/EC.00100-14

Zhang J, Mao Z, Xue W, Li Y, Tang G, Wang A, Zhang Y, Wang H (2011) $K u 80$ gene is related to non-homologous end-joining and genome stability in Aspergillus niger. Curr Microbiol 62:1342-1346. doi:10. 1007/s00284-010-9853-5 